\title{
Subdural Hematoma as a Complication of Hemostatic Deficiency Secondary to Liver Cirrhosis
}

-Report of Two Cases-

\author{
Tomoo FURUI, Akihiro YAMADA and Kinjiro IwaTA
}

Department of Neurological Surgery, Aichi Medical University, Aichi

\begin{abstract}
We describe two cases of subdural hematoma as a complication of thrombocytopenia and coagulation deficiency due to liver cirrhosis. Both patients were treated surgically despite uncorrected hemostatic deficiency. There have been no reports of similar cases in the literature.
\end{abstract}

Key words: subdural hematoma, hemostatic deficiency, liver cirrhosis

\section{Introduction}

Nontraumatic subdural hematoma is known to occur occasionally in association with bleeding diatheses, such as thrombocytopenic purpura, ${ }^{3)}$ disseminated intravascular coagulopathy (DIC) due to malignancy, ${ }^{2)}$ hemophilia, ${ }^{7}$ and complications of hemodialysis. ${ }^{4)}$ We present two cases in which subdural hematoma developed as a complication of hemostatic deficiency due to liver cirrhosis. To our knowledge, no similar cases have been previously described.

\section{Case Reports}

Case 1: A 74-year-old male suddenly developed headache and nausea followed by several episodes of vomiting on the morning of May, 1987, after which he visited a local physician. Analgesics were prescribed and he returned home. That evening, he was found unconscious by his family and was brought to our clinic. On admission, he was comatose and exhibited anisocoria (right $<$ left), right hemiparesis, and the Babinski reflex bilaterally. Computed tomography (CT) demonstrated a crescentshaped area of high density in the left frontotemporoparietal subdural region and a marked midline shift to the right (Fig. 1 upper).

Laboratory examinations yielded the following data: white blood cell (WBC) count, $16,000 / \mathrm{mm}^{3}$;

Received October 20, 1988; Accepted March 29, 1989
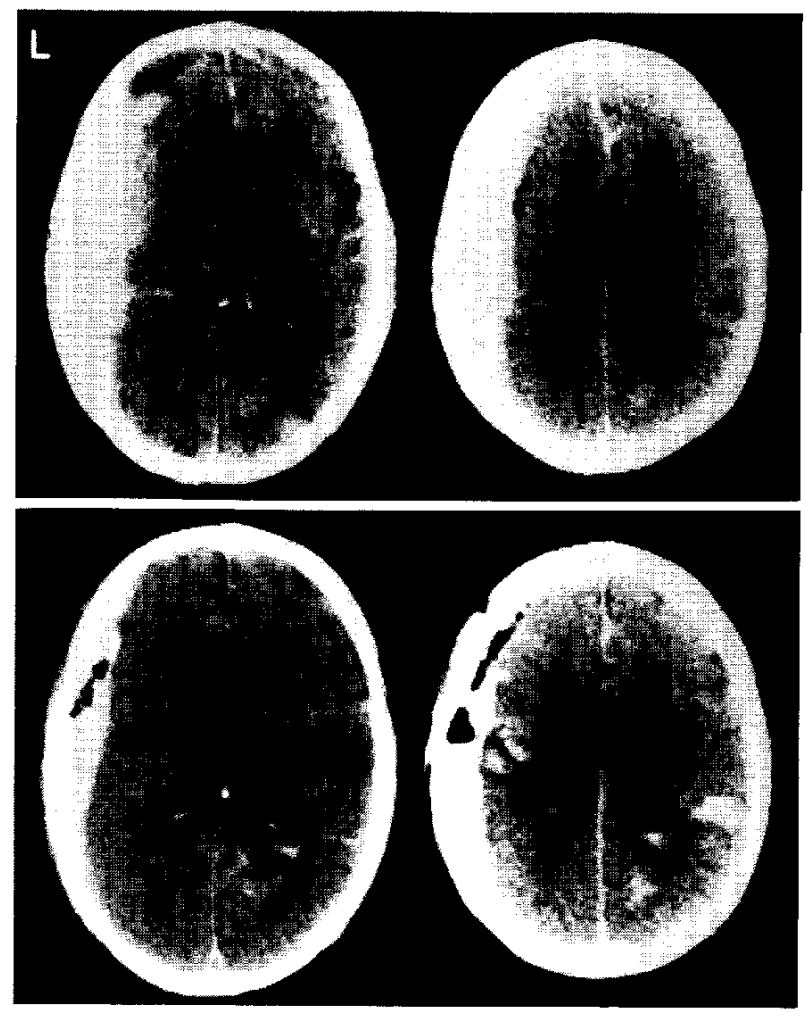

Fig. 1 Case 1. CT scans obtained on admission (upper) and 6 days after burr hole craniotomy (lower). The subdural hematoma, shown as high density over the left hemisphere, has diminished and the midline shift has disappeared. 
red blood cell (RBC) count, $2.49 \times 10^{6} / \mathrm{mm}^{3}$; hemoglobin, $7.0 \mathrm{gm} / \mathrm{dl}$; hematocrit, $22.3 \%$; platelet count, $43,000 / \mathrm{mm}^{3}$; prothrombin time (PT), $16.2 \mathrm{sec}$ onds (control, 12.5 seconds); partial thromboplastin time (PTT), 31.5 seconds (control, 30.9 seconds); plasma fibrinogen, $87 \mathrm{mg} / \mathrm{dl}$ (normal, $200-400 \mathrm{mg}$ / dl); and serum fibrinogen degradation product (FDP), $45 \mu \mathrm{g} / \mathrm{ml}$ (normal, $<5 \mu \mathrm{g} / \mathrm{ml}$ ).

His history included no recent head trauma. He had been diagnosed as having liver cirrhosis secondary to chronic hepatitis about 5 years previously and had been managed by a local physician. Laboratory data obtained 1 year prior to this admission were as follows: WBC count, $3000 / \mathrm{mm}^{3} ; \mathrm{RBC}$ count, $3.36 \times 10^{6} / \mathrm{mm}^{3}$; hemoglobin, $9.9 \mathrm{gm} / \mathrm{dl}$; hematocrit, $28.6 \%$; platelet count, $92,000 / \mathrm{mm}^{3}$; total serum protein, $7.1 \mathrm{gm} / \mathrm{dl}$ (albumin, $52.8 \% ; \gamma$ globulin, 26.4\%); glutamic-oxaloacetic transaminase (GOT), 38 IU (normal, 11-25 IU); glutamic-pyruvic transaminase (GPT), 22 IU (normal, 5-20 IU); cholinesterase, $0.35 \Delta \mathrm{pH}$ (normal, $0.70-1.17 \Delta \mathrm{pH}$ ); thymol turbidity test (TTT), 7.7 U (normal, <4.0 U); and zinc sulfate turbidity test, 21.2 U (normal, 3-12 $U$ ). PT was slightly prolonged at 12.7 seconds (control, 12.0 seconds). Other coagulation data were within normal limits. ${ }^{99 \mathrm{~m}} \mathrm{Tc}$-scintigraphy showed moderate splenomegaly.

Four hours after admission, two burr holes were drilled urgently under local anesthesia. Because of the bleeding diathesis, a standard large craniotomy was avoided, although the onset of subdural hematoma was acute. Approximately $150 \mathrm{ml}$ of hematoma, partly clotted, were evacuated by suction and subdural drainage was instituted for 6 days. Fresh blood was transfused during surgery.

He regained consciousness, and postoperative CT showed the subdural hematoma to have been removed (Fig. 1 lower). The platelet count and coagulation data improved to near normal after additional transfusion of fresh blood and platelets. Unfortunately, he died of respiratory insufficiency on the 53rd day of hospitalization. An autopsy was not permitted, but postoperative bone marrow examination disclosed no malignancy. Microscopic examination of the dura specimens removed through the burr holes also showed no malignancy.

Case 2: A 58-year-old female had been treated for liver cirrhosis complicated by esophageal varices and gastric ulcer since January, 1983, at which time she had visited our hospital for evaluation of hematemesis. On the evening of July, 1984, she displayed abnormal behavior, including walking naked about her home, and then became drowsy. She was brought to our clinic and diagnosed as being in hepatic coma on the basis of elevation of serum ammonia to $310 \mu \mathrm{g} / \mathrm{dl}$ (normal, 20-60 $\mu \mathrm{g} / \mathrm{dl}$ ) and triphasic waves on her electroencephalogram. She regained consciousness 2 days later, when the ammonia level dropped.

Laboratory data at this time were as follows: WBC count, $3400 / \mathrm{mm}^{3}$; RBC count, $3.36 \times 10^{6} / \mathrm{mm}^{3}$; hemoglobin, $12.7 \mathrm{gm} / \mathrm{dl}$; hematocrit, $37.1 \%$; platelet count, $68,000 / \mathrm{mm}^{3} ; \mathrm{PT}, 14.3$ seconds (control, 30.4 seconds); PTT, 49.1 seconds (control, 30.4 seconds); fibrinogen, $168 \mathrm{mg} / \mathrm{dl}$; FDP, $10 \mu \mathrm{g} / \mathrm{ml}$; total protein, $6.7 \mathrm{gm} / \mathrm{dl}$ (albumin, 50.2\%; $\gamma$-globulin, $42.2 \%$ ); GOT, 57 IU; GPT, 32 IU; lactate dehydrogenase, 470 IU (normal, 116-299 IU); cholinesterase, $0.22 \Delta \mathrm{pH}$; and TTT, $18.5 \mathrm{U}$.

The following December, she was again hospitalized. She complained of headache and became lethargic, although her serum ammonia level was normal. She was transferred to our department the next day. There had been no episode of head trauma. Neurological examination revealed anisocoria (right $>$ left) and left hemiparesis with the Babinski reflex. A CT scan showed a crescent-shaped area of heterogeneous density in the right frontotem-
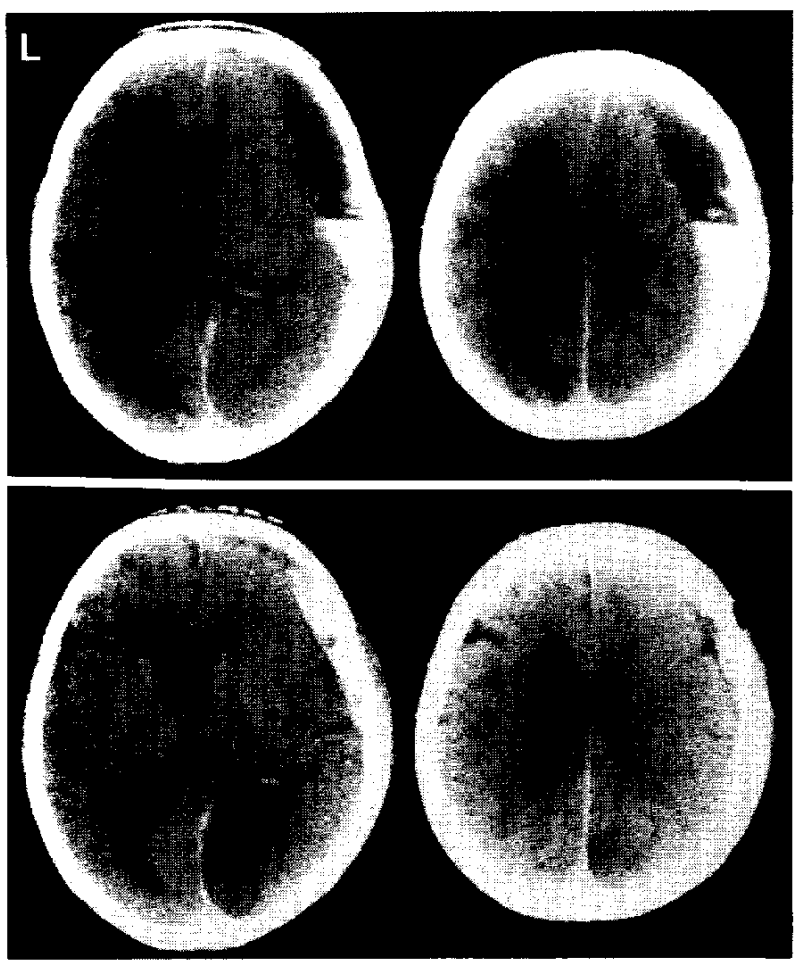

Fig. 2 Case 2. CT scans obtained on admission to our department (upper) and 5 days after bur hole craniotomy (lower). The midline shift has disappeared by draining the hematoma. 
poroparietal subdural region (Fig. 2 upper). The laboratory data were as follows: WBC count, 4900/ $\mathrm{mm}^{3}$; RBC count, $3.31 \times 10^{6} / \mathrm{mm}^{3}$; hemoglobin, $11.6 \mathrm{gm} / \mathrm{dl}$; hematocrit, $35.2 \%$; platelet count, $89,000 / \mathrm{mm}^{3}$; PT, 17.3 seconds (control, $12.8 \mathrm{sec}$ onds); PTT, 47.4 seconds (control, 29.5 seconds); fibrinogen, $175 \mathrm{mg} / \mathrm{dl}$; and FDP, $20 \mu \mathrm{g} / \mathrm{ml}$.

Through emergency two burr holes, about 130 ml of dark brown fluid was drained. Subdural drainage was continued for 5 days. Fresh blood and platelets were transfused during and after surgery.

Postoperatively, she improved neurologically and showed partial recovery of hemostatic function (Fig. 2 lower). However, her consciousness remained cloudy and she died of pulmonary complications 38 days after admission. Postmortem examination showed no malignancy in any organ, including the liver and the dura mater.

\section{Discussion}

Liver cirrhosis is well known to induce hemostatic deficiency. The incidence of liver cirrhosis is reportedly higher in patients with intracerebral hemorrhage than in normal population. ${ }^{1)}$ Because of the small number of patients with subdural hematoma, however, these authors were unable to document a significant relationship between liver cirrhosis and subdural hematoma. To our knowledge, there have been no published reports of such association, although it would seem plausible that subdural hematoma might develop as a complication of hemostatic deficiency due to liver cirrhosis. Therefore, we felt it worthwhile to report these two cases.

The hemostatic deficiency in hepatocellular dysfunction is ascribed to thrombocytopenia and coagulation abnormalities. The former is believed to be caused by hypersplenism, while the latter has been attributed to a deficiency of fibrinogen and/or increased fibrinolysis. Hyperfibrinolysis is thought to result from impaired hepatic clearance of plasminogen activators and reduced synthesis of fibrinolytic inhibitors. The deficiency of coagulation factors is known to be due to reduction of their synthesis; relatively recently, it was suggested that an increase in their consumption through intravascular coagulation may also be involved. Some patients with hepatocellular disease have laboratory findings suggestive of DIC, and these are occasionally reversed by heparin administration. ${ }^{8)}$

Both of our patients had marked thrombocytopenia, prolongation of both PT and PTT, increased serum FDP, and decreased plasma fibrinogen. These suggest but do not conclusively prove DIC. The diagnosis of DIC is difficult in cases of liver cirrhosis because hyperfibrinolysis, hypofibrinogenemia, and thrombocytopenia may all be present in hepatocellular disease, without intravascular coagulation. In fact, the preoperative laboratory abnormalities in our two cases do not fulfill the criteria for a diagnosis of DIC in the presence of liver cirrhosis, ${ }^{5)}$ although they would nearly satisfy the criteria in the absence of hepatic dysfunction. ${ }^{6}$ ) However, these cases do illustrate that coagulation deficiency and/ or thrombocytopenia due to liver cirrhosis can play a significant role in the development of nontraumatic subdural hematoma.

In addition to pointing out this association, these cases provide tentative guidelines for the surgical management of acute subdural hematoma in the presence of hemostatic deficiency. Large craniotomy is commonly performed for acute subdural hematoma, and is reportedly practicable in cases with uncorrected bleeding diathesis. ${ }^{10)}$ However, it is preferable to use a less invasive procedure, such as burr hole craniotomy or needle puncture ${ }^{9)}$ under local anesthesia, provided that the results are equivalent to those achievable with large craniotomy. Both of our patients showed neurological improvement and survived more than 1 month after burr hole opening and continuous subdural drainage, until respiratory complications developed. Complete evacuation of even a partially clotted hematoma was possible with this procedure.

In conclusion, coagulation deficiency and/or thrombocytopenia due to liver cirrhosis should be considered as a cause, or at least a precipitating factor, of nontraumatic subdural hematoma. In such cases, surgery need not be ruled out.

\section{References}

1) Boudouresques G, Hauw J-J, Meininger V, Escourolle R, Pertuiset B, Buge A, Lhermitte F, Castaigne P: Hepatic cirrhosis and intracranial hemorrhage: Significance of the association in 53 pathological cases. Ann Neurol 8: 204-205, 1980

2) Furui $T$, Ichihara $K$, Ikeda $A$, Inao $S$, Hirai $N$, Yoshida J, Kageyama N: Subdural hematoma associated with disseminated intravascular coagulation in patients with advanced cancer. $J$ Neurosurg 58: 398401, 1983

3) Kolluri VRS, Reddy DR, Reddy PK, Naidu MRC, Kumari CS: Subdural hematoma secondary to immune thrombocytopenic purpura: Case report. Neurosurgery 19: 635-636, 1986

4) Leonard A, Shapiro FL: Subdural hematoma in regularly hemodialyzed patients. Ann Intern Med 82: $650-658,1975$ 
5) Minna JD, Robboy SJ, Colman RW: Disseminated Intravascular Coagulation in Man. Springfield, CC Thomas, 1974, pp 3-18

6) Minna JD, Robboy SJ, Colman RW: Disseminated Intravascular Coagulation in Man. Springfield, CC Thomas, 1974, pp 160-166

7) Olsen ER: Intracranial surgery in hemophiliacs. Arch Neurol 21: 401-412, 1969

8) Ratnoff OD: Disordered hemostasis in hepatic disease, in Schiff L, Schiff ER (eds): Disease of the Liver, ed 6. Philadelphia, Lippincott, 1987, pp 187207

9) Verlooy P, Lamers BJM, de Haan GJ, Noach LA:
Successful treatment of acute subdural haematoma associated with severe bleeding disorder. J Neurol 234: 254-256, 1987

10) Winston $K$, Conner S: Successful evacuation of subdural hematoma in the presence of severe coagulopathy. Neurosurgery 11: 277-279, 1982

Address reprint requests to: T. Furui, M.D., Department of Neurological Surgery, Aichi Medical University, 21 Karimata, Yazako, Nagakute-cho, Aichi-gun, Aichi 480-11, Japan. 